\title{
EGF-induced urokinase plasminogen activator receptor promotes epithelial to mesenchymal transition in human gastric cancer cells
}

\author{
PINGPING WANG $^{1}$, MAOYUAN MA $^{2}$ and SHANHUI ZHANG ${ }^{1}$ \\ Departments of ${ }^{1}$ Clinical Laboratory and ${ }^{2}$ Gastroenterology, Taizhou Second People's Hospital, \\ Taizhou, Jiangsu 225599, P.R. China
}

Received March 2, 2017; Accepted August 10, 2017

DOI: $10.3892 /$ or.2017.5920

\begin{abstract}
Epidermal growth factor (EGF) signaling has been shown to induce epithelial to mesenchymal transition (EMT) in many types of cancer cells. However, the molecular mechanism of EGF-induced EMT in gastric cancer remains largely unknown. In the present study, we found that human gastric cancer cell lines SGC-7901 and BGC-823 underwent EMT phenotypic changes upon exposure to EGF. The induction of EMT was consistent with aggressive characteristics such as increased cell migration, invasion and clonogenic growth. Additionally, EGF stimulation also led to the upregulation of urokinase plasminogen activator receptor (UPAR) both at mRNA and protein levels. Knockdown of uPAR by siRNA significantly attenuated EMT induction by EGF in SGC-7901 and BGC-823 cells. Furthermore, EGF increased ERK1/2 activity and blocking ERK1/2 signaling with its inhibitor, U0126, markedly inhibited EGF-induced uPAR expression and consequently EMT. Collectively, the present study demonstrated that EGF induced aggressiveness of gastric cancer cells by activating EMT, which involved the activation of the ERK1/2 pathway and, subsequently, uPAR expression.
\end{abstract}

\section{Introduction}

Epithelial to mesenchymal transition (EMT), which is defined by the loss of epithelial characteristics and the acquisition of mesenchymal properties, has been found to contribute to cancer progression and metastasis in multiple types of cancer including gastric cancer (1-3). EMT phenotype in cancers has also been associated with poor clinical outcome $(2,4)$. Moreover, it has been proposed that signaling pathways involved in metastasis are shared by EMT $(5,6)$. Therefore,

Correspondence to: Professor Shanhui Zhang, Department of Clinical Laboratory, Taizhou Second People's Hospital, 27 Jiankang Road, Jiangsu 225599, P.R. China

E-mail: shanhuizhang@126.com

Key words: EGF, ERK1/2, uPAR, EMT elucidation of the signaling pathways that govern EMT may advance our understanding of the mechanisms of tumor metastasis.

EMT is believed to be governed by signals from the tumor microenvironment including a variety of cytokines and growth factors, such as epidermal growth factor (EGF) and transformation growth factor- $\beta$ (TGF- $\beta$ ) (7). An in vivo study by Goswami et al (8) has suggested that macrophages express EGF, which promotes the formation of elongated protrusions and cell invasion of carcinoma cells. The capacity of EGF to induce EMT was previously reported in various cell models including gastric cancer cells $(9,10)$. However, the molecular mechanisms underlying the induction of EMT by EGF are still not well characterized.

The urokinase plasminogen activator receptor (uPAR), a glycosyl phosphatidylinositiol-anchored receptor, has been implicated in EGF signaling and cancer invasion (11-13). It has been demonstrated that an increased level of UPAR was essential to the induction of EMT, and this increase was correlated to tumor progression and aggressiveness $(14,15)$. The synthesis of uPAR was increased by diverse growth factors including EGF $(11,16,17)$. Mounting evidence has suggested that extracellular signal-regulated kinase $1 / 2($ ERK1/2) is a potent modulator of uPAR expression in cancer cells $(18,19)$. In addition, a study by Tushir and D'Souza-Schorey (20) also revealed that ERK1/2 regulated UPAR expression during HGF-induced tubule development. Furthermore, previous studies have indicated that UPAR played a pivotal role in promoting EGF-induced tumor invasion $(16,19)$. Based on the aforementioned findings and the lack of mechanistic studies in establishing the role of EGF-induced upregulation of UPAR with respect to the acquisition of EMT and tumor cell aggressiveness, we used gastric cancer cells as a preclinical model for the present study. The results in the present study indicated that EGF-induced EMT involved a cascade of signaling events including activation of ERK1/2 signaling and subsequent upregulation of uPAR.

\section{Materials and methods}

Cell culture and treatment. Human gastric cancer cell lines, BGC-823 and SGC-7901, were purchased from the Chinese 
Academy of Sciences Cell Bank (Shanghai, China); all cell lines were maintained at $37^{\circ} \mathrm{C}$ in a $5 \% \mathrm{CO}_{2}$ incubator in Dulbecco's modified Eagle's medium (DMEM) supplemented with $10 \%$ fetal bovine serum (FBS), $100 \mathrm{U} / \mathrm{ml}$ penicillin and $100 \mu \mathrm{g} / \mathrm{ml}$ streptomycin. EGF was added to DMEM supplemented with $1 \%$ FBS at a final concentration of $10 \mathrm{ng} / \mathrm{ml}$. Cells were made quiescent by serum starvation overnight followed by EGF treatment for 7 days before experiments were conducted.

Wound healing assay. Cells were treated with or without EGF for 7 days. Then, the cells were plated into a 96-well plate. When cells were $95-100 \%$ confluent, wounding was performed by scraping the cell monolayer with a $10-\mu 1$ pipette tip. Wound closure was monitored by visual examination under an inverted microscope with an 100X objective, at time-point zero and after $24 \mathrm{~h}$.

Matrigel invasion assay. Cells were treated with or without EGF for 7 days. Then $5 \times 10^{3}$ cells in DMEM with $1 \%$ phosphatebuffered saline (PBS) were seeded onto the upper chamber, which were coated with Matrigel (Sigma-Aldrich, St. Louis, MO, USA). As a chemoattractant, DMEM with $10 \%$ FBS was added into the lower compartment. After incubation for $24 \mathrm{~h}$, the cells were fixed in methanol for $20 \mathrm{~min}$ and stained with $0.1 \%$ crystal violet for $20 \mathrm{~min}$. The cells on the upper surface of the filter were wiped off with a cotton swab and the number of cells that had migrated out to the lower surface of the membranes was counted in 5 randomly selected fields. The experiment was repeated at least 3 times independently.

Colony formation assay. Cells were treated with or without EGF for 7 days. Subsequently, the cells were plated at a density of $2 \times 10^{3}$ cells in 6-well plates. Then the cells were incubated at $37^{\circ} \mathrm{C}$ for 14 days. Next, colonies were stained with $2 \%$ crystal violet, and the number of colonies that consisted of $>20$ cells was counted.

Small interfering RNA (siRNA) transfection. siRNA-specific for uPAR was purchased from GenePharma (Shanghai, China). As a non-specific control siRNA, scrambled siRNA duplex was used which was also purchased from GenePharma. The sequences of siRNA for uPAR were: 1,5'-GGUGACGCCUUC AGCAUGAdTdT-3'; 2, 5'-GCCGUUACCUCGAAUGCAUdT dT-3'; 3,5'-CACCACCAAAUGCAACGAGdTdT-3'; and for the scrambled sequence: 5'-UUCUCCGAACGUGUCACGUTT-3'. Transfection was carried out using Lipofectamine 2000 (Invitrogen, Carlsbad, CA, USA) following the manufacturer's instructions. Silencing of uPAR was assayed at the mRNA and protein expression level at $48 \mathrm{~h}$ after transfection.

Western blotting. Cells were harvested after the indicated treatment. Protein was extracted in RIPA lysis buffer. Fifty micrograms of protein was loaded on an SDS-PAGE gel, followed by protein separation and electroblotting onto a polyvinylidene difluoride (PVDF) membrane. The membrane was labeled with the following primary antibodies: mouse anti-E-cadherin, mouse anti-vimentin and goat anti-uPAR (Santa Cruz Biotechnology, Santa Cruz, CA, USA), rabbit anti-ERK1/2 and anti-phospho-ERK1/2 (Thr202/Tyr204) (Cell Signaling Technology, Boston, MA, USA), and mouse
anti-GAPDH antibody (Chemicon, Temecula, CA, USA). HRP-conjugated secondary antibodies were incubated in 5\% BSA in Tris-buffered saline with Tween-20 (TBST) buffer for $2 \mathrm{~h}$ at room temperature. Immunoreactivity was detected using an enhanced chemiluminescence detection system (Pierce, Rockford, IL, USA).

$R T-P C R$. Total RNAs were isolated using TRIzol reagent according to the manufacturer's protocol (Invitrogen). Then, cDNA was synthesized using the SuperScript First Strand Synthesis System (Invitrogen), and amplified by polymerase chain reaction (PCR) using the following primers: GAPDH, 5'-TGAACGGGAAGCTCACTGG-3' (sense) and 5'-TCCACC ACCCTGTTGCTGTA-3' (antisense); E-cadherin, 5'-AGGAT GGCTGAAGGTGACAGAG-3' (sense) 5'-TGGCCTCAAAA TCCAAGCCC-3' (antisense); vimentin, 5'-GATGTGGATGT TTCCAAGCC-3' (sense) 5'-ACCAGAGGGAGTGAATC CAG-3' (antisense); uPAR, 5'-TTACCGAGGTTGTGTGTG GG-3' (sense) 5'-GGGCATGTTGGCACATTGAG-3' (antisense). The PCR for GAPDH was performed in 26 cycles at $95^{\circ} \mathrm{C}$ for $30 \mathrm{sec}, 55^{\circ} \mathrm{C}$ for $30 \mathrm{sec}$, and $72^{\circ} \mathrm{C}$ for $30 \mathrm{sec}$, for E-cadherin in 28 cycles at $95^{\circ} \mathrm{C}$ for $30 \mathrm{sec}, 56^{\circ} \mathrm{C}$ for $30 \mathrm{sec}$, and $72^{\circ} \mathrm{C}$ for $40 \mathrm{sec}$, for vimentin in 28 cycles at $95^{\circ} \mathrm{C}$ for $30 \mathrm{sec}$, $55^{\circ} \mathrm{C}$ for $30 \mathrm{sec}$, and $72^{\circ} \mathrm{C}$ for $40 \mathrm{sec}$, and for uPAR in $28 \mathrm{cycles}$ at $95^{\circ} \mathrm{C}$ for $30 \mathrm{sec}, 55^{\circ} \mathrm{C}$ for $30 \mathrm{sec}$, and $72^{\circ} \mathrm{C}$ for $30 \mathrm{sec}$. The PCR products were resolved by electrophoresis on $1 \%$ agarose.

Immunofluorescence staining. Cells were treated with or without EGF for 7 days and grown on cover slips for $24 \mathrm{~h}$. The cells on the slips were washed with PBS, fixed with $4 \%$ paraformaldehyde, and permeabilized with $0.2 \%$ Triton X-100. The cells were then incubated with anti-phospho-ERK1/2 (Thr202/Tyr204) antibodies for $2 \mathrm{~h}$ followed by PBS washes. Subsequently, the cells were incubated with Rhodamineconjugated anti-rabbit antibody for $1.5 \mathrm{~h}$. The cells were finally mounted with anti-fade mounting medium and viewed using a Leica DM2500 fluorescence microscope (Leica, Wetzlar, Germany).

Statistical analysis. Statistical analysis was carried out using the SPSS software (SPSS, Inc., Chicago, IL, USA). Student's t-test was used to analyze the differences between 2 groups. When comparisons between multiple groups were carried out, one-way ANOVA followed by SNK tests were employed. Statistical significance was considered at $\mathrm{p}<0.05$.

\section{Results}

EGF induces EMT in gastric cancer cells. EGF is one of the most abundant growth factors found in the tumor microenvironment and induces EMT in multiple types of cancer cells (21-23). In an attempt to recapitulate the in vivo situation where cells are chronically exposed to EGF in the tumor microenvironment, we exposed SGC-7901 and BGC-823 cells to $10 \mathrm{ng} / \mathrm{ml}$ EGF for up to 1 week. Following 7 days of exposure to EGF, the morphologies of SGC-7901 and BGC-823 cells were found to be completely changed to a mesenchymal phenotype, with elongated and disseminated appearances (Fig. 1A). To confirm the mesenchymal phenotype, we assessed the expression of molecular markers of EMT such as vimentin 
A

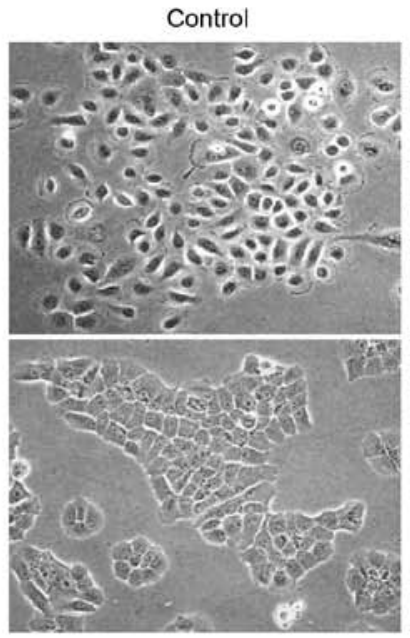

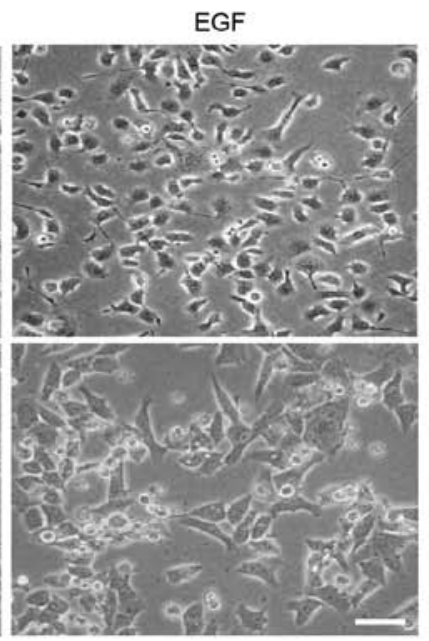

SGC-7901

BGC-823
B

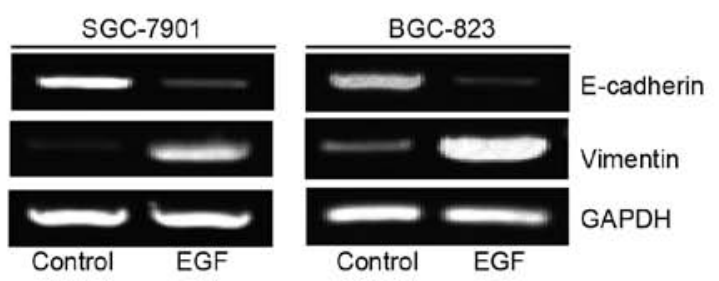

C

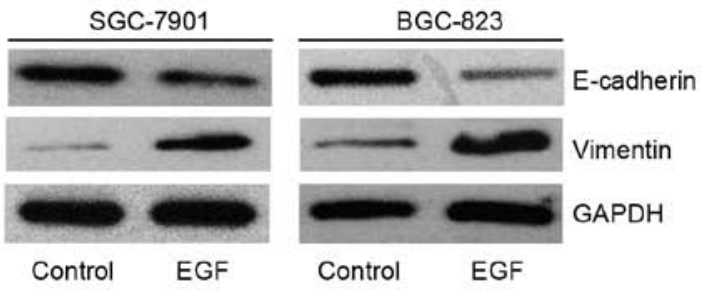

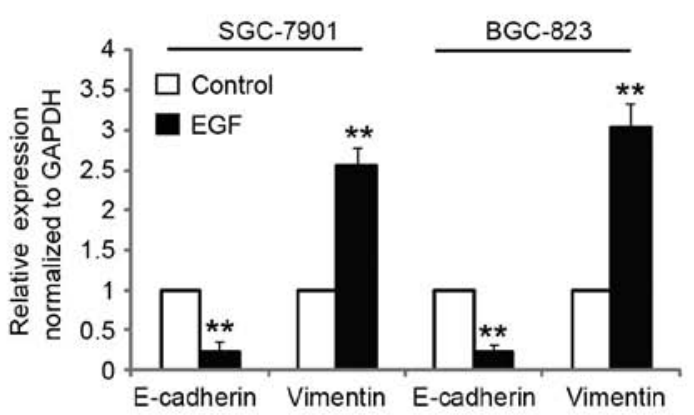

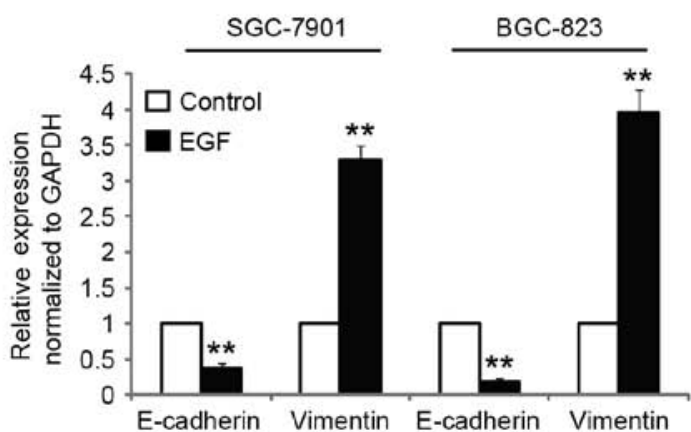

Figure 1. Induction of EMT in gastric cancer cells by exposure to EGF. EGF $(10 \mathrm{ng} / \mathrm{ml})$ was added to SGC-7901 and BGC-823 cells in culture media and maintained for 7 days with the medium changed every $24 \mathrm{~h}$ with freshly added EGF. (A) Detectable morphological conversion was observed from EGF treatment. Images of the cells were captured by phase-contrast microscope. Scale bar, $100 \mu \mathrm{m}$. (B) Endogenous mRNA levels of E-cadherin and vimentin were analyzed by RT-PCR. (C) Endogenous protein levels of E-cadherin and vimentin were analyzed by western blotting; ${ }^{*}$ p $<0.05$ in the cultures with EGF relative to the cultures without EGF. EMT, epithelial to mesenchymal transition; EGF, epidermal growth factor.

and found that the mRNA and protein levels were increased after EGF treatment (Fig. 1B and C). In addition, the expression of E-cadherin, an epithelial marker, was downregulated after EGF treatment (Fig. 1B and C). Collectively, these data revealed that EGF induced the SGC-7901 and BGC-823 cells to undergo EMT-like phenotypic changes.

Cell migration and invasive characteristics are increased in gastric cancer cells after EGF treatment. It is well known that tumor cells with an EMT phenotype are more motile and invasive (24). Therefore, we examined the migratory and invasive capacity of SGC-7901 and BGC-823 cells in response to EGF treatment. Our results revealed that the migration rate was increased with the treatment of cells with EGF, as compared with the control cells (Fig. 2A). Using a Boyden chamber invasion assay with Matrigel-coated polycarbonate membranes, we found that more cells incubated with EGF had migrated through the membrane than the control cells (Fig. 2B). We next determined whether EGF promoted the clonogenic growth of SGC-7901 and BGC-823 cells by colony formation assay. Treatment of cells with EGF resulted in a significant promotion of clonogenic growth (Fig. 2C). Collectively, these results demonstrated that EGF-induced EMT was accompanied by enhanced cell migration, invasion and clonogenic growth.

EGF induces EMT via activation of the ERK1/2 pathway. In order to assess the mechanism by which EGF treatment induced EMT, we focused our investigation on ERK1/2 signaling since it has been implicated in EMT induction, 

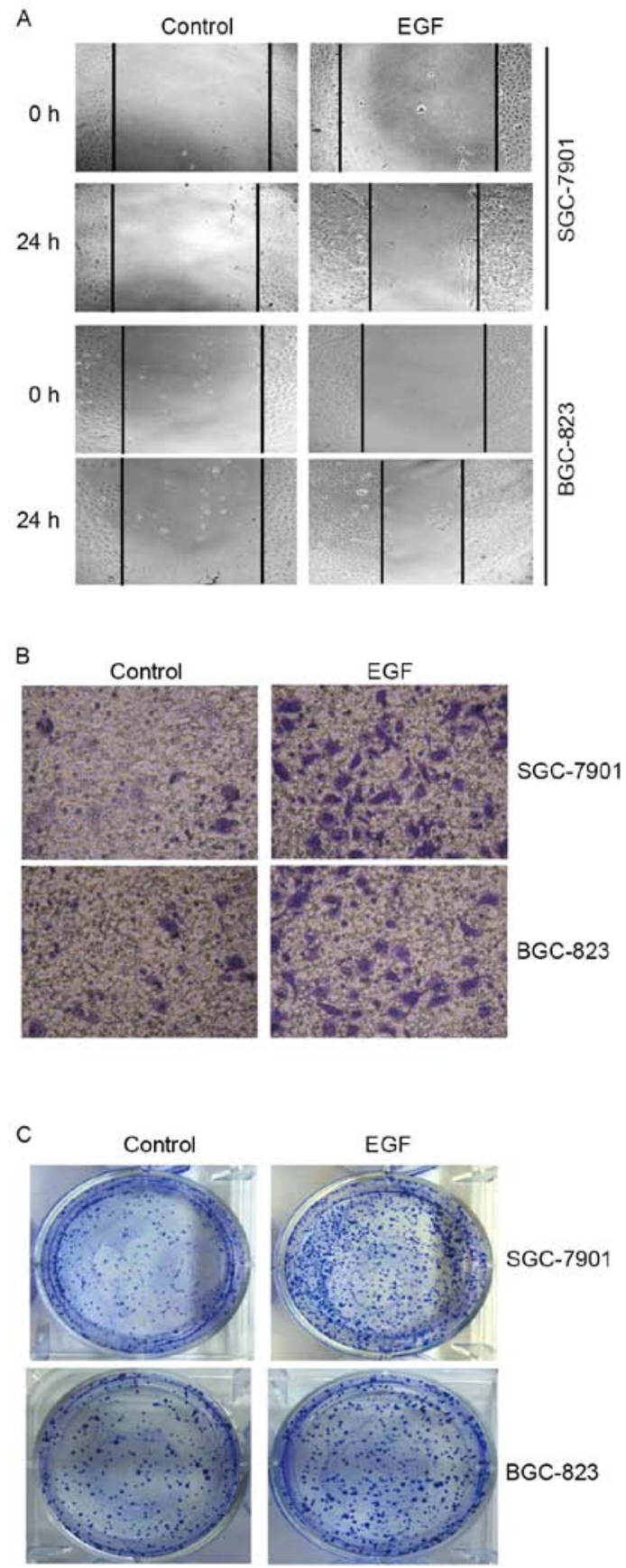
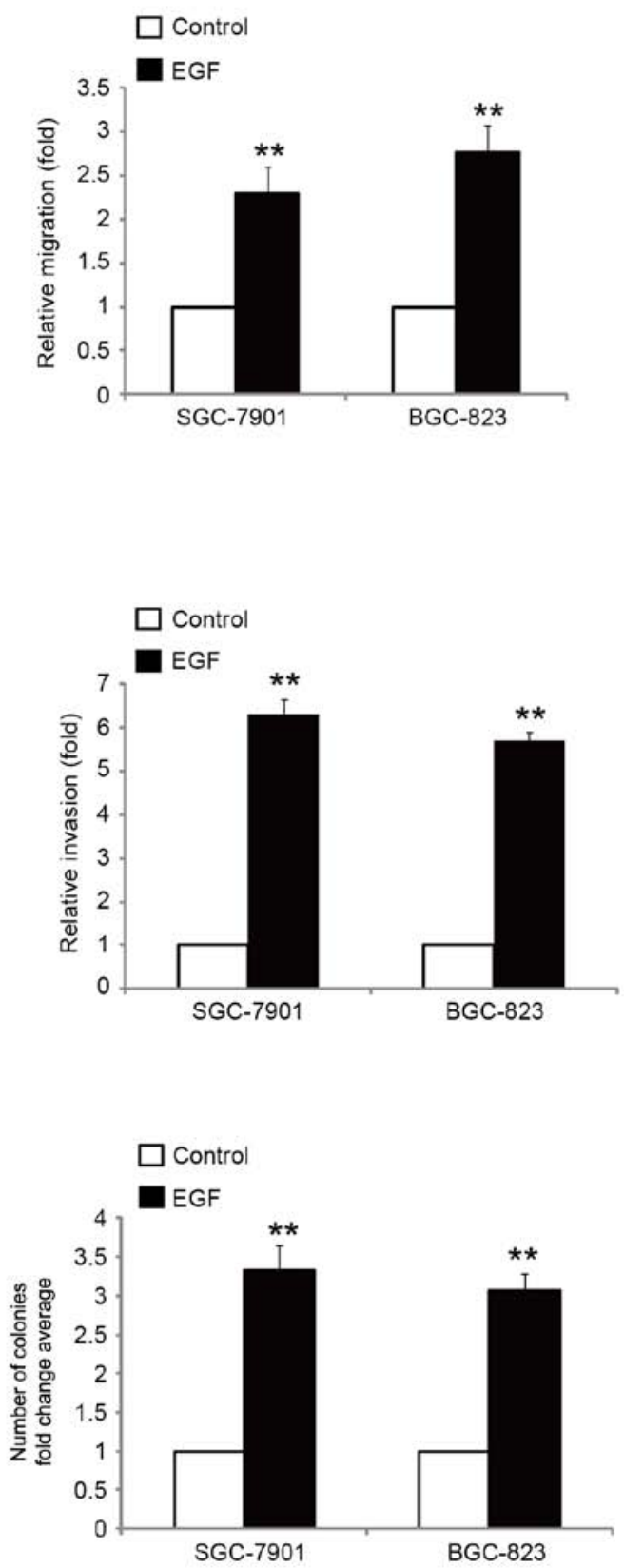

Figure 2. SGC-7901 and BGC-823 cells exhibit a significant increase in cell migration, invasive and tumorigenic characteristics upon EGF treatment. EGF was added to SGC-7901 and BGC-823 cells in culture media and maintained for 7 days with the medium changed every $24 \mathrm{~h}$ with freshly added EGF. (A) The relative cell migration rate was determined using wound closure assay. (B) Cell invasion was assessed using the Matrigel invasion assay. (C) Colony formation assay results with its quantitative analysis; " ${ }^{* *}<0.05$ in the cultures with EGF relative to the cultures without EGF. EGF, epidermal growth factor.

metastasis and invasion (25-27). We found that the level of phospho-ERK1/2 was significantly increased after EGF stimulation, whereas the total protein level of ERK1/2 remained unaltered (Fig. 3A). The results revealed that pretreatment of SGC-7901 and BGC-823 cells with ERK1/2 inhibitor prior to treatment with EGF maintained epithelial morphology, while cells treated with EGF revealed transformation to mesenchymal morphology (Fig. 3B). Moreover, treatment of SGC-7901 and BGC-823 cells with ERK1/2 inhibitor exhibited partial reversal, where we observed incomplete attenuation of EMT phenotype, as documented by the decreased expression of vimentin, and increased expression of epithelial marker E-cadherin (Fig. 3C). These results revealed that EGF-induced EMT is mediated by the activation of ERK1/2.

Since ERK1/2 regulates EMT not only depending on its phosphorylation status, but also on its redistribution to the nucleus and plasma membrane (28), we also examined p-ERK1/2 localization in cultured cells after EGF treatment. Immunofluorescence staining revealed that p-ERK1/2 was weak and localized in the cytoplasm and nucleus of control cells. However, p-ERK1/2 abundance was obviously increased in the nucleus and periphery of the cells after exposure to EGF (Fig. 4). 
A
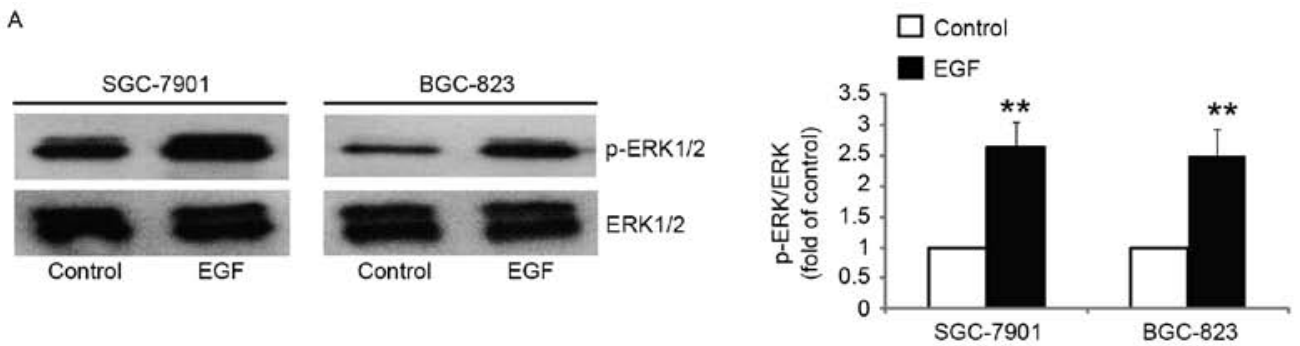

B
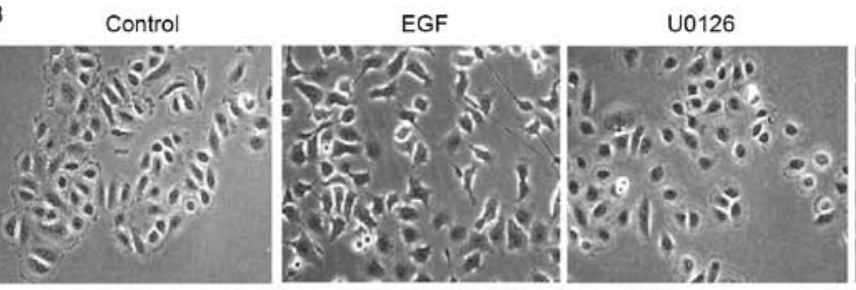

EGF+U0126
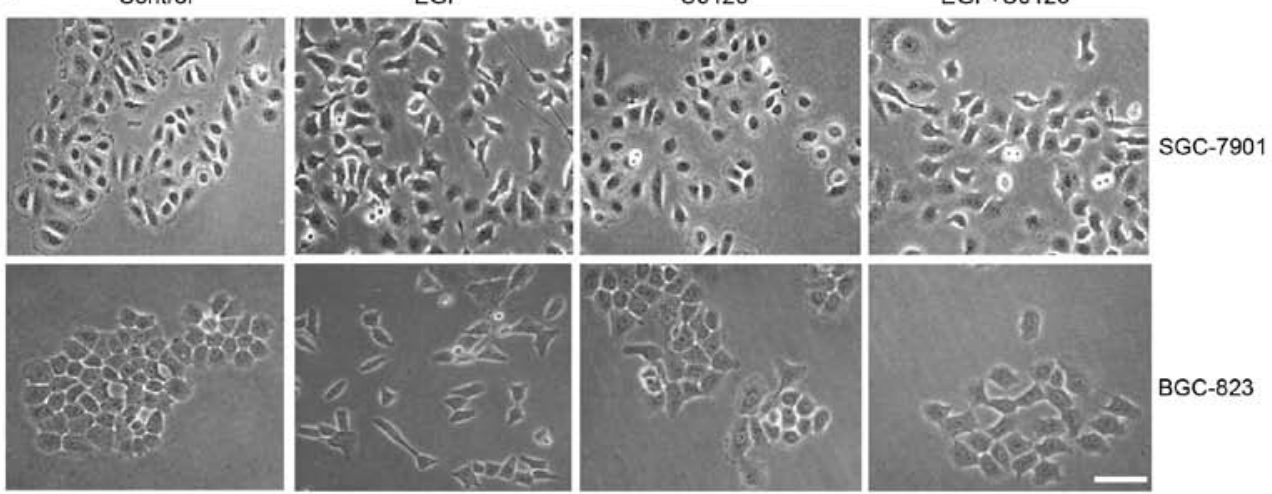

C

SGC-7901
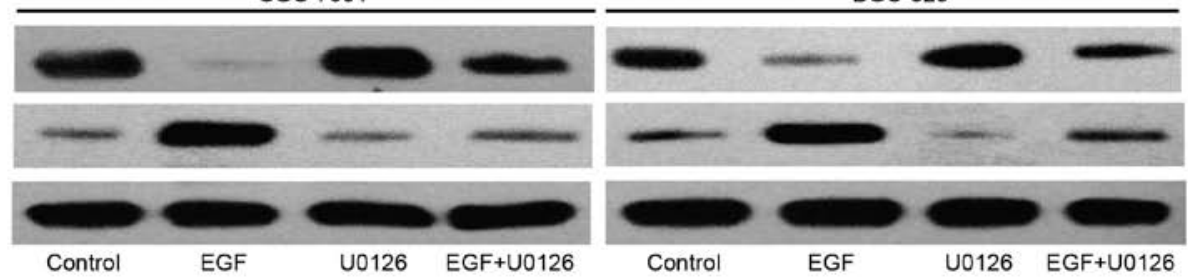

E-cadherin

Vimentin

GAPDH

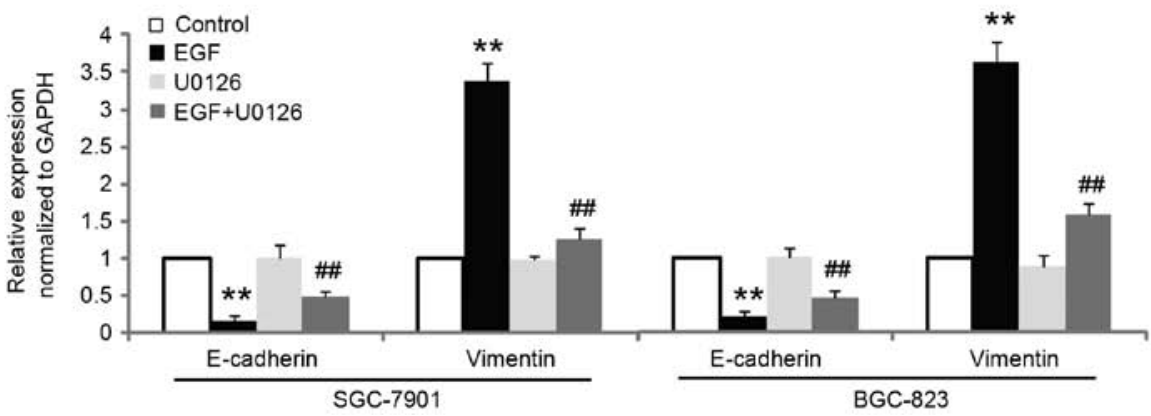

Figure 3. Activation of the ERK1/2 pathway is required for EGF-induced EMT. (A) Activation of ERK1/2 by EGF. SGC-7901 and BGC-823 cells were treated with EGF $(10 \mathrm{ng} / \mathrm{ml})$ for 7 days, and the media was changed every $24 \mathrm{~h}$. Phosphorylation of ERK1/2 at Thr202/Tyr204 was determined as described in the 'Materials and methods'. (B) The effect of ERK1/2 inhibitor on EGF-induced morphological conversion. SGC-7901 and BGC-823 cells were pretreated with ERK inhibitor U0126 $(10 \mu \mathrm{M})$ before EGF treatment. The images of the cells were captured by phase-contrast microscope. Scale bar, $100 \mu \mathrm{m}$. (C) The effect of the ERK inhibitor on the protein levels of E-cadherin and vimentin. SGC-7901 and BGC-823 cells were pretreated with ERK inhibitor U0126 (10 $\mu \mathrm{M})$ before EGF treatment, and the endogenous protein levels of E-cadherin and vimentin were analyzed using western blotting; ${ }^{* *} \mathrm{p}<0.05$, compared with the control;

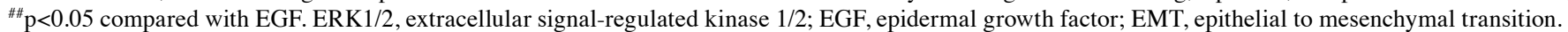

UPAR is required for EGF-induced EMT. Accumulating evidence has indicated that UPAR in cancer cells promote EMT $(14,29)$. We then wished to examine whether uPAR is required for EGF-induced EMT in gastric cancer cells. Notably, we found a dramatic increase in the expression of uPAR both at the mRNA and protein levels in SGC-7901 and BGC-823 cells after EGF stimulation (Fig. 5A and B). In order to further investigate the role of UPAR in EGF-induced EMT in gastric cancer cells, we knocked down the expression of UPAR protein in SGC-7901 and BGC-823 cells by uPAR-specific
siRNA. The uPAR-siRNA transfection resulted in significant knockdown of uPAR expression as shown by western blotting (Fig. 5C) and significantly attenuated EGF-induced EMT, which was confirmed morphologically (Fig. 5D). The transfection of SGC-7901 and BGC-823 cells with uPAR-siRNA led to the partial reversal of the EMT phenotype as documented by the decreased expression of vimentin, and the increased expression of E-cadherin (Fig. 5E). These results demonstrated that UPAR upregulation by EGF is mechanistically linked with EGF-induced EMT in gastric cancer cells. 

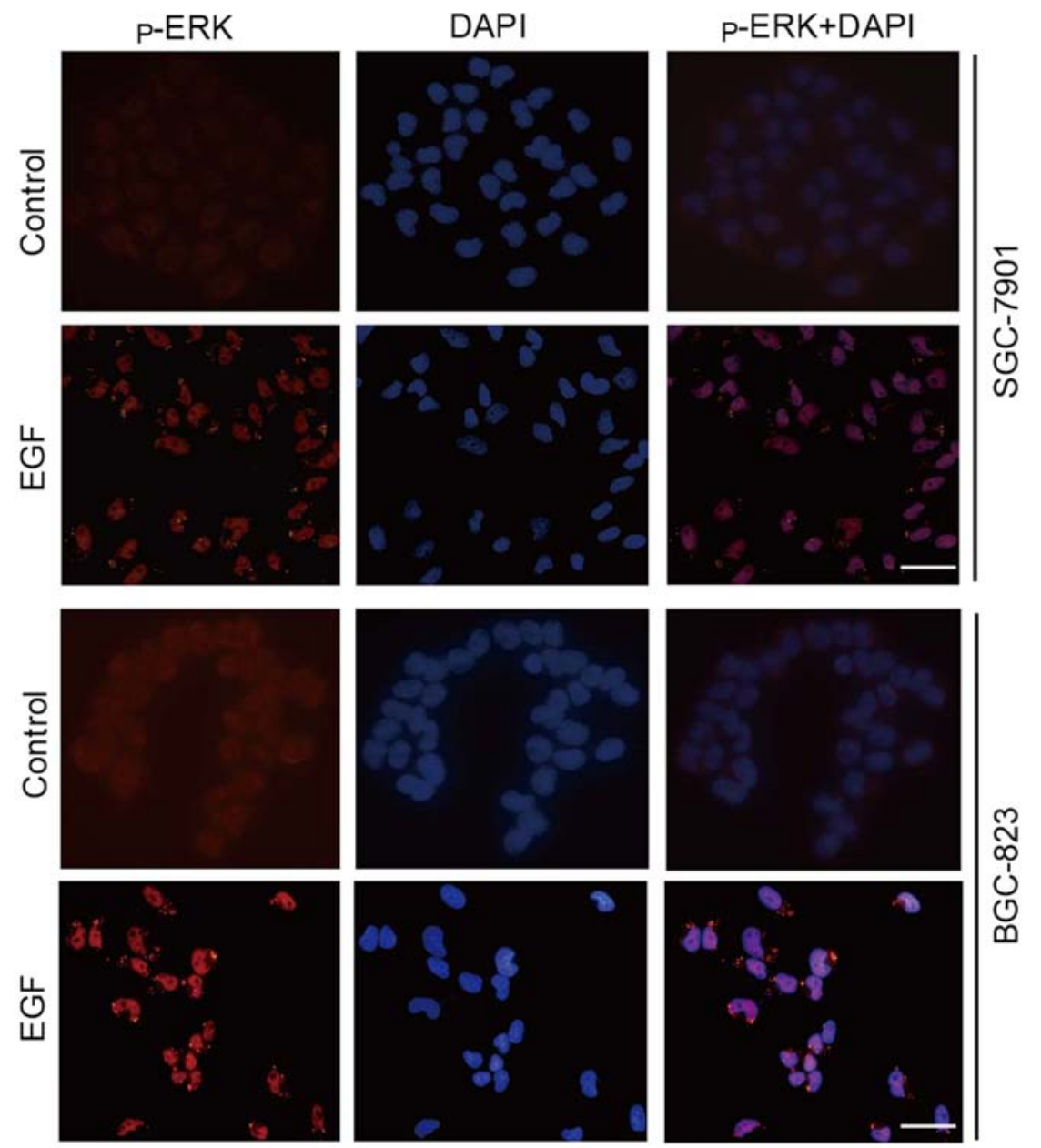

Figure 4. EGF stimulates nuclear translocation of p-ERK1/2 in gastric cancer cells. After treatment with EGF for 7 days, the expression of p-ERK (red) in SGC-7901 and BGC-823 cells was examined by immunofluorescence microscopy as described in the 'Materials and methods'. The cells were counter-stained with DAPI (blue). Scale bar, $100 \mu \mathrm{m}$. EGF, epidermal growth factor.

UPAR acts as a downstream target of ERK1/2 and mediates $E G F$-induced EMT. It has been well documented that ERK1/2 regulates growth factor-induced uPAR expression in cancer cells $(16,19)$. To determine whether the induced expression of uPAR by EGF observed in our systems was ERK1/2-dependent, we blocked ERK1/2 activity by pretreating the cells with U0126 and examined uPAR expression after stimulation with EGF. The results revealed that pretreatment with $10 \mu \mathrm{M}$ U0126 significantly inhibited EGF-induced uPAR expression in comparison with control cells (Fig. 6A). However, inhibition of uPAR expression did not alter the EGF-induced increase of ERK1/2 phosphorylation (Fig. 6B). These results revealed that uPAR acts as a downstream molecule of ERK $1 / 2$ and mediates EGF-induced EMT.

\section{Discussion}

EGF, which can be directly produced by tumor-associated stroma cells, is one of the most abundant growth factors found in tumor microenvironment and acts in a paracrine fashion to cause EMT in different types of solid tumors, including gastric cancer (28,30-32). EGF treatment has also been demonstrated to increase cultured cancer cell migration, invasion and proteolytic activity (33-35). In an attempt to recapitulate the in vivo situation where cells are chronically exposed to EGF in the tumor microenvironment, we exposed gastric cancer cells to EGF for up to one week. In addition, in order to understand the principal effects of EGF in the absence of other growth factors, we intended to use serum-free medium for gastric cancer cells to exclude other unnecessary growth factors in the serum. However, this starvation culture induces apoptosis of SGC-7901 and BGC-823 cells after 3-4 days (data not shown). Therefore, EGF was added to the medium supplemented with $1 \%$ FBS, which did not cause apoptosis within one week. In our system, gastric cancer cell lines (SGC-7901 and BGC-823) underwent EMT phenotypic changes after chronic exposure to EGF, which was consistent with the decreased expression of an epithelial marker concomitant with the increased expression of mesenchymal markers. In order to further characterize these cells, we assessed the cell migration, invasion and tumorigenic potential of EGF-treated cells compared to the control cells. Our data revealed increased cell migration, invasion and tumorigenic potential of EGF-treated cells compared to the control cells. Therefore, EGF induced EMT in gastric cancer cells, which is a critical step for tumor invasion and metastasis. Based on this, the signaling mechanisms underlying the effect of EGF on the induction of EMT were investigated. 
A

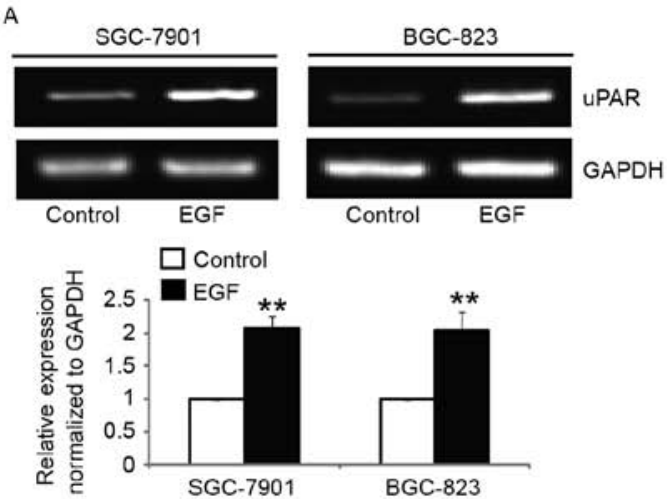

B
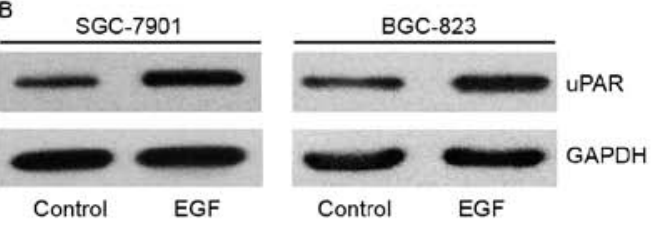

$\square$ Control

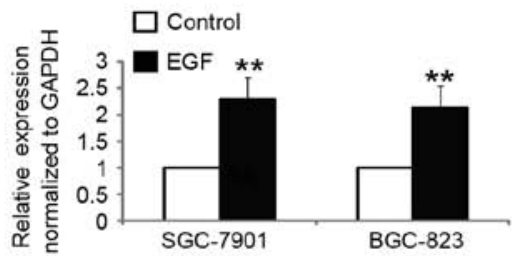

C
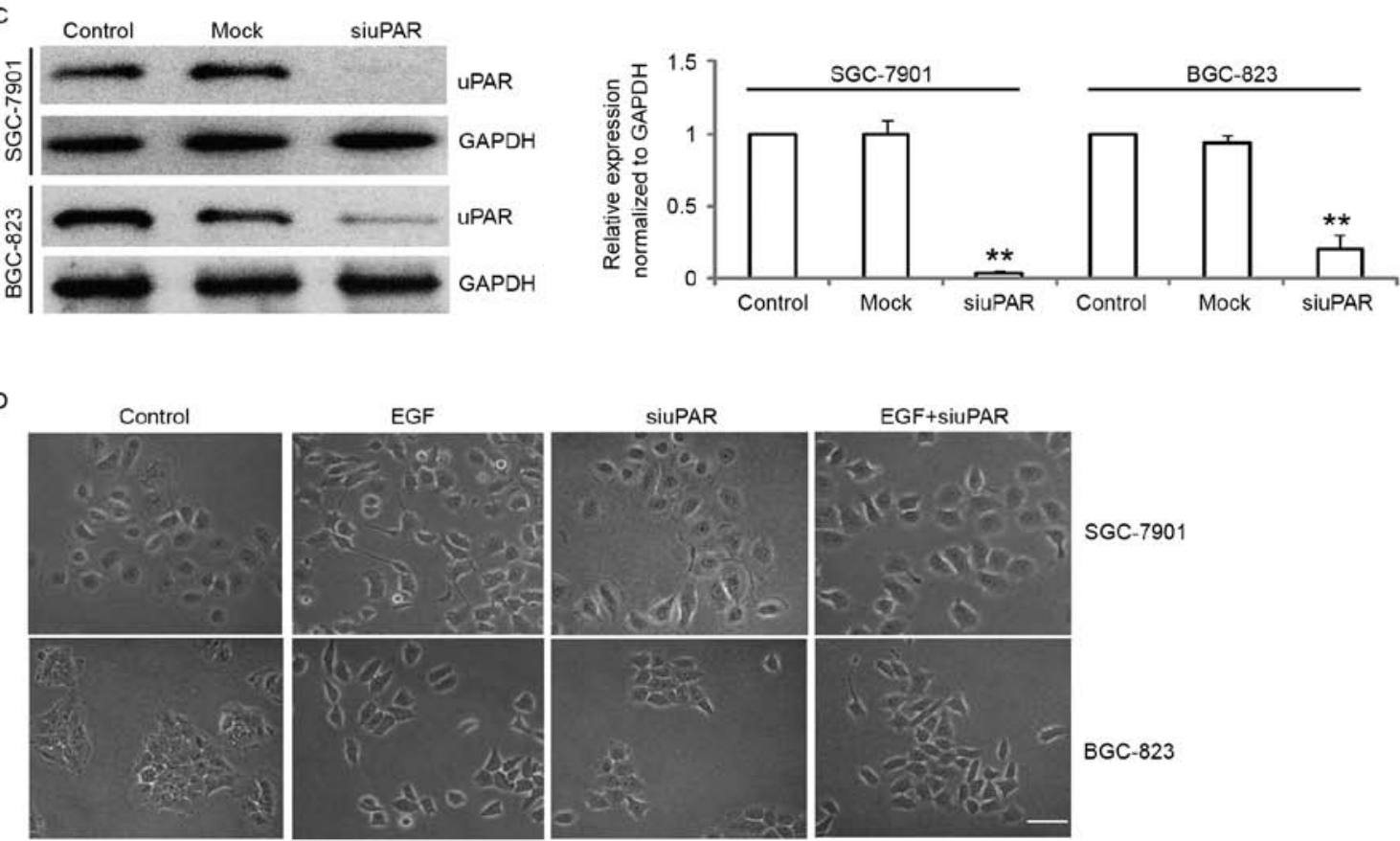

SGC-7901

E

SGC-7901
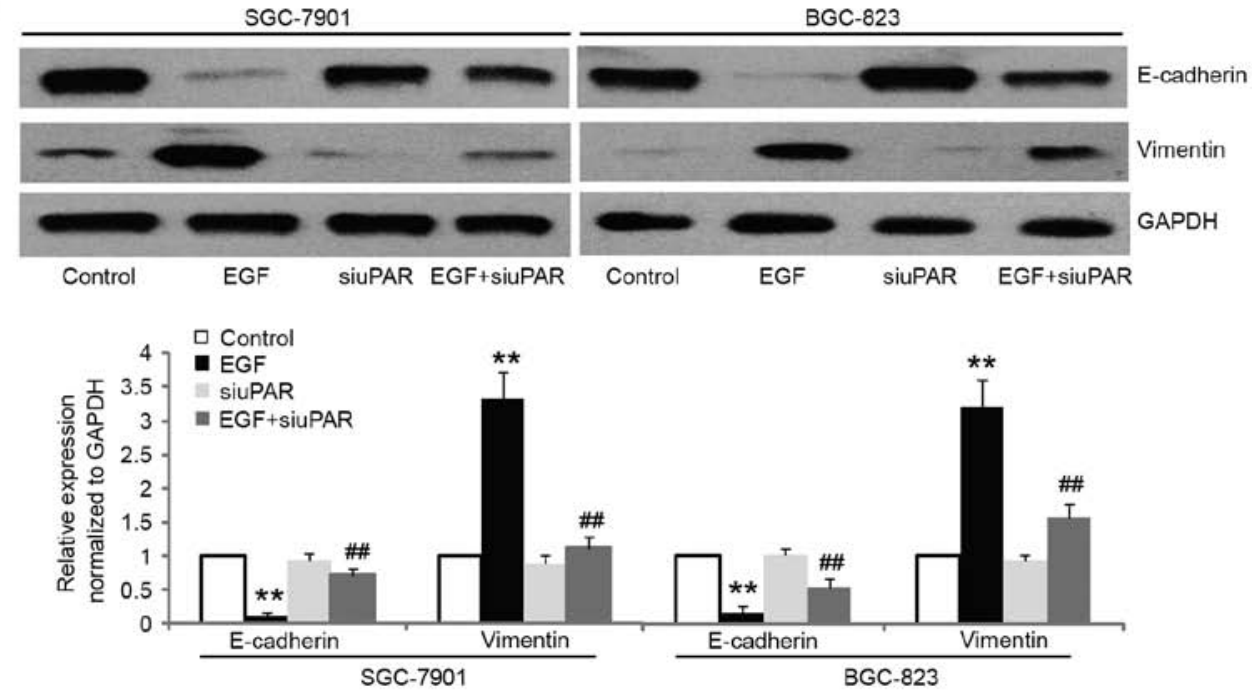

Figure 5. EGF-induced EMT occurred by upregulation of uPAR. (A and B) Upregulation of uPAR by EGF. SGC-7901 and BGC-823 cells were treated with EGF $(10 \mathrm{ng} / \mathrm{ml})$ for 7 days, and the media was changed every $24 \mathrm{~h}$. The endogenous mRNA and protein levels of uPAR were assayed by (A) RT-PCR and (B) western blotting, respectively. (C) The effect of siRNA on the intracellular levels of uPAR. Total protein extracts from SGC-7901 and BGC-823 cells transfected with siRNA-uPAR (si-uPAR) or scrambled siRNA (mock) were analyzed by western blotting for uPAR. GAPDH was used as a loading control. (D) Effect of si-uPAR on EGF-induced morphological conversion. Images of the cells were captured by phase-contrast. Scale bar, $100 \mu \mathrm{m}$. (E) The effect of si-uPAR on the protein levels of E-cadherin and vimentin. SGC-7901 and BGC-823 cells were transfected with si-uPAR, and then treated with EGF for 7 days. The endogenous protein levels of E-cadherin and vimentin were analyzed by western blotting; ${ }^{* *} \mathrm{p}<0.05$, compared with the control; ${ }^{\# \# ~} \mathrm{p}<0.05$ compared with EGF. EGF, epidermal growth factor; EMT, epithelial to mesenchymal transition; uPAR, urokinase plasminogen activator receptor. 


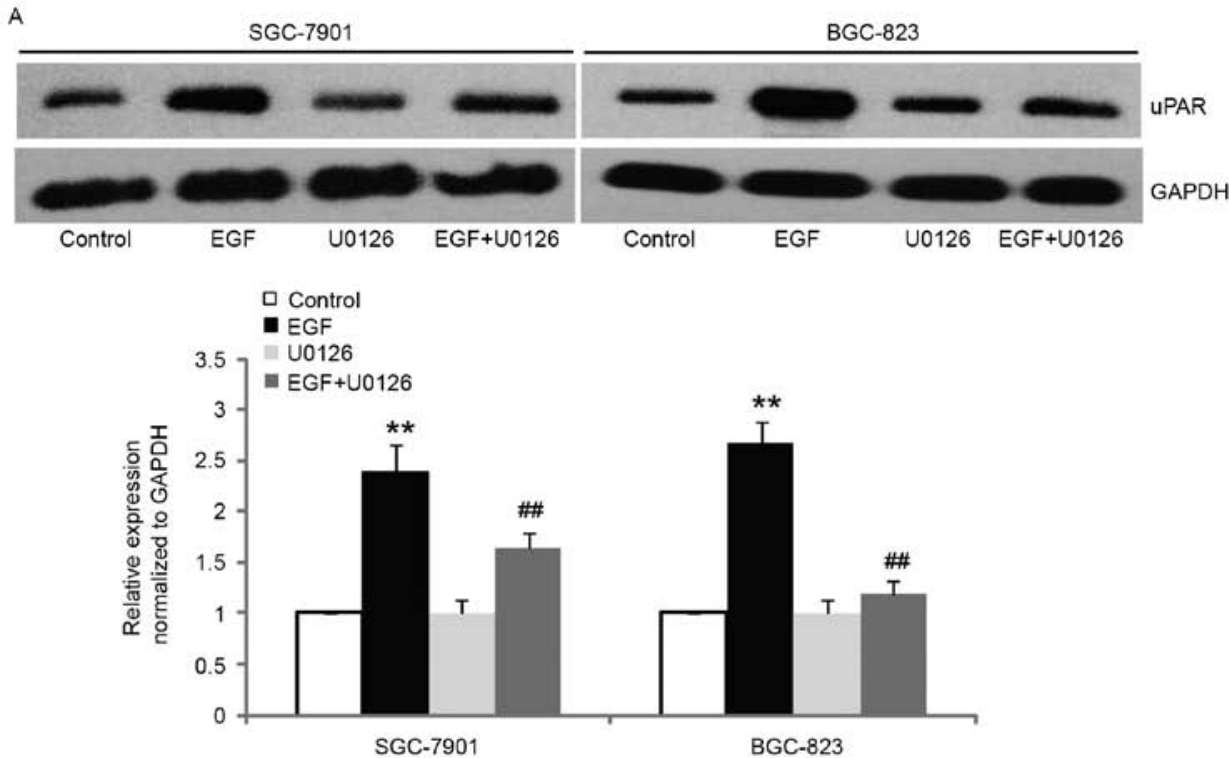

B
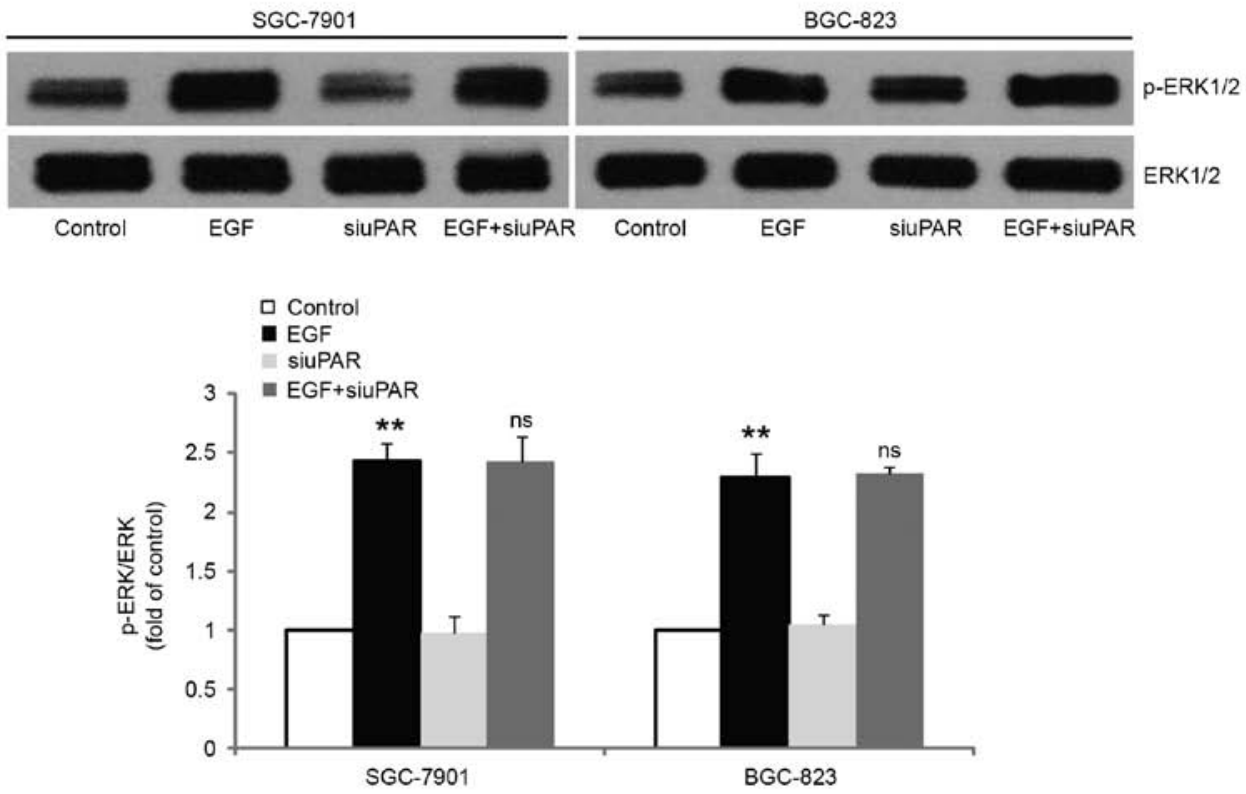

Figure 6. EGF upregulates uPAR by activation of the ERK1/2 pathway. (A) EGF-mediated upregulation of uPAR requires ERK1/2 activation. SGC-7901 and BGC-823 cells were pretreated with ERK1/2 inhibitor U0126 $(10 \mu \mathrm{M})$ before EGF treatment, and the endogenous protein level of uPAR was assayed by western blotting. (B) EGF-mediated ERK1/2 activation is uPAR-independent. SGC-7901 and BGC-823 cells were transfected with si-uPAR, and then treated with EGF for 7 days. The phosphorylation of ERK at Thr202/Tyr204 was determined by western blotting; ${ }^{* *} \mathrm{p}<0.05$, compared with the control; ${ }^{* \# p}<0.05$, compared with EGF; NS, no statistical significance compared with EGF. EGF, epidermal growth factor; uPAR, urokinase plasminogen activator receptor; ERK1/2, extracellular signal-regulated kinase 1/2.

There is increasing evidence that the activation of ERK1/2 signaling contributes to cancer invasion and metastasis $(36,37)$. ERK1/2 signaling has also been implicated in EMT induced by growth factors, such as TGF- $\beta 1$ and FGF $(38,39)$. Similar to these findings, our results revealed that the activity of ERK $1 / 2$ was increased after EGF treatment. Inhibition of ERK activity by U0126 significantly prevented EGF-induced EMT, suggesting that EGF-induced ERK activation was responsible for the induction of EMT.

Increased expression of UPAR in human types of cancer is associated with metastasis, whereas in low-grade cancer, forced expression of uPAR promotes tumor metastasis $(40,41)$. Previous studies have revealed that endogenous uPAR plays an important regulatory role in EMT and that EGF-induced cell invasion is mediated by the upregulation of uPAR expression in gastric cancer cells $(14,16)$. In the present study, we found that EGF-induced EMT was associated with an increase in UPAR expression. Knockdown of UPAR by uPAR specific siRNA significantly attenuated EMT induction by EGF treatment. These results revealed that EGF-induced EMT was mediated by upregulation of UPAR.

In the present study, our data demonstrated that in gastric cancer cells, ERK1/2 and uPAR mediated the EGF-induced EMT. In some types of cells, uPAR is a downstream target of the ERK1/2 signaling cascade and inhibition of ERK1/2 was sufficient to suppress uPAR expression $(19,42,43)$. In the present study, we demonstrated that blocking ERK1/2 activity significantly prevented EGF-induced uPAR expression. 
Previous studies have demonstrated that ERK1/2 activity was regulated by uPAR $(44,45)$. We found that specific downregulation of UPAR in gastric cancer cells did not alter EGF-induced ERK1/2 activation. Therefore, it is possible that EGF induces uPAR expression via the ERK1/2 pathway and, in turn, stimulates initiation of EMT. The different results obtained by different study groups may be due to the different cell systems used and receptor-coupled signaling in these studies.

In summary, the present study demonstrated that treatment with EGF induced cell migration and invasion by activating EMT in gastric cancer cells. EGF treatment can lead to the activation of the ERK1/2/uPAR cascade in gastric cancer cells and contribute to EMT. These findings elucidate a molecular pathway linking EGF signaling with uPAR in governing EMT, cell motility and invasiveness, which may represent a rational molecular target for manipulating gastric cancer.

\section{References}

1. Brabletz T: EMT and MET in metastasis: Where are the cancer stem cells? Cancer Cell 22: 699-701, 2012.

2. Franco-Chuaire ML, Magda Carolina SC and Chuaire-Noack L: Epithelial-mesenchymal transition (EMT): Principles and clinical impact in cancer therapy. Invest Clin 54: 186-205, 2013.

3. Fantozzi A, Gruber DC, Pisarsky L, Heck C, Kunita A, Yilmaz M Meyer-Schaller N, Cornille K, Hopfer U, Bentires-Alj M, et al: VEGF-mediated angiogenesis links EMT-induced cancer stemness to tumor initiation. Cancer Res 74: 1566-1575, 2014.

4. No authors listed: An oncogenic splice variant drives EMT and metastasis in breast cancer. Cancer Discov 3: OF16, 2013.

5. Chang RM, Xu JF, Fang F, Yang H and Yang LY: MicroRNA-130b promotes proliferation and EMT-induced metastasis via PTEN/p-AKT/HIF-1 $\alpha$ signaling. Tumour Biol 37: 10609-10619, 2016.

6. Sannino G, Armbruster N, Bodenhöfer M, Haerle U, Behrens D, Buchholz M, Rothbauer U, Sipos B and Schmees C: Role of BCL9L in transforming growth factor- $\beta$ (TGF- $\beta$ )-induced epithelial-to-mesenchymal-transition (EMT) and metastasis of pancreatic cancer. Oncotarget 7: 73725-73738, 2016.

7. Gao D, Vahdat LT, Wong S, Chang JC and Mittal V: Microenvironmental regulation of epithelial-mesenchymal transitions in cancer. Cancer Res 72: 4883-4889, 2012.

8. Goswami S, Sahai E, Wyckoff JB, Cammer M, Cox D, Pixley FJ, Stanley ER, Segall JE and Condeelis JS: Macrophages promote the invasion of breast carcinoma cells via a colony-stimulating factor-1/epidermal growth factor paracrine loop. Cancer Res 65 : 5278-5283, 2005.

9. Luo BH, Xiong F, Wang JP, Li JH, Zhong M, Liu QL, Luo GQ, Yang XJ, Xiao N, Xie B, et al: Epidermal growth factorlike domain-containing protein 7 (EGFL7) enhances EGF receptor-AKT signaling, epithelial-mesenchymal transition, and metastasis of gastric cancer cells. PLoS One 9: e99922, 2014.

10. Muthusami S, Prabakaran DS, Yu JR and Park WY: EGF-induced expression of Fused Toes Homolog (FTS) facilitates epithelialmesenchymal transition and promotes cell migration in ME180 cervical cancer cells. Cancer Lett 351: 252-259, 2014.

11. Henic E, Noskova V, Høyer-Hansen G, Hansson S and Casslén B: Estradiol attenuates EGF-induced rapid uPAR mobilization and cell migration via the G-protein-coupled receptor 30 in ovarian cancer cells. Int J Gynecol Cancer 19: 214-222, 2009.

12. Hu J, Jo M, Cavenee WK, Furnari F, VandenBerg SR and Gonias SL: Crosstalk between the urokinase-type plasminogen activator receptor and EGF receptor variant III supports survival and growth of glioblastoma cells. Proc Natl Acad Sci USA 108 : 15984-15989, 2011.

13. Hu J, Muller KA, Furnari FB, Cavenee WK, VandenBerg SR and Gonias SL: Neutralizing the EGF receptor in glioblastoma cells stimulates cell migration by activating uPAR-initiated cell signaling. Oncogene 34: 4078-4088, 2015.

14. Gupta R, Chetty C, Bhoopathi P, Lakka S, Mohanam S, Rao JS and Dinh DE: Downregulation of uPA/uPAR inhibits intermittent hypoxia-induced epithelial-mesenchymal transition (EMT) in DAOY and D283 medulloblastoma cells. Int J Oncol 38: 733-744, 2011.
15. Ashour AA, Gurbuz N, Alpay SN, Abdel-Aziz AA, Mansour AM, Huo L and Ozpolat B: Elongation factor-2 kinase regulates TG2/\$1 integrin/Src/uPAR pathway and epithelial-mesenchymal transition mediating pancreatic cancer cells invasion. J Cell Mol Med 18: 2235-2251, 2014.

16. Baek MK, Kim MH, Jang HJ, Park JS, Chung IJ, Shin BA, Ahn BW and Jung YD: EGF stimulates uPAR expression and cell invasiveness through ERK, AP-1, and NF- $\kappa \mathrm{B}$ signaling in human gastric carcinoma cells. Oncol Rep 20: 1569-1575, 2008.

17. Smith HW and Marshall CJ: Regulation of cell signalling by uPAR. Nat Rev Mol Cell Biol 11: 23-36, 2010.

18. Bessard A, Frémin C, Ezan F, Coutant A and Baffet G: MEK/ERK-dependent UPAR expression is required for motility via phosphorylation of P70S6K in human hepatocarcinoma cells. J Cell Physiol 212: 526-536, 2007.

19. Hu Z, Xu R, Liu J, Zhang Y, Du J, Li W, Zhang W, Li Y, Zhu Y and Gu L: GEP100 regulates epidermal growth factor-induced MDA-MB-231 breast cancer cell invasion through the activation of Arf6/ERK/uPAR signaling pathway. Exp Cell Res 319: 1932-1941, 2013.

20. Tushir JS and D'Souza-Schorey C: ARF6-dependent activation of ERK and Racl modulates epithelial tubule development. EMBO J 26: 1806-1819, 2007.

21. Li J, Shan F, Xiong G, Chen X, Guan X, Wang JM, Wang WL, $\mathrm{Xu} \mathrm{X}$ and Bai Y: EGF-induced C/EBP $\beta$ participates in EMT by decreasing the expression of miR-203 in esophageal squamous cell carcinoma cells. J Cell Sci 127: 3735-3744, 2014.

22. Wang Y, Lin Z, Sun L, Fan S, Huang Z, Zhang D, Yang Z, Li J and Chen W: Akt/Ezrin Tyr353/NF- $\kappa$ B pathway regulates EGF-induced EMT and metastasis in tongue squamous cell carcinoma. Br J Cancer 110: 695-705, 2014

23. Cordonnier T, Bishop JL, Shiota M, Nip KM, Thaper D, Vahid S, Heroux D, Gleave M and Zoubeidi A: Hsp27 regulates $\mathrm{EGF} / \beta$-catenin mediated epithelial to mesenchymal transition in prostate cancer. Int J Cancer 136: E496-E507, 2015.

24. Savagner P: Epithelial-mesenchymal transitions: From cell plasticity to concept elasticity. Curr Top Dev Biol 112: 273-300, 2015.

25. Ha GH, Park JS and Breuer EK: TACC3 promotes epithelialmesenchymal transition (EMT) through the activation of PI3K/Akt and ERK signaling pathways. Cancer Lett 332: 63-73, 2013.

26. Chen B, Zeng X, He Y, Wang X, Liang Z, Liu J, Zhang P, Zhu H, $\mathrm{Xu} \mathrm{N}$ and Liang S: STC2 promotes the epithelial-mesenchymal transition of colorectal cancer cells through AKT-ERK signaling pathways. Oncotarget 7: 71400-71416, 2016.

27. Wang Z, Qu L, Deng B, Sun X, Wu S, Liao J, Fan J and Peng Z: STYK1 promotes epithelial-mesenchymal transition and tumor metastasis in human hepatocellular carcinoma through MEK/ERK and PI3K/AKT signaling. Sci Rep 6: 33205, 2016.

28. Zhang Y, Du J, Zheng J, Liu J, Xu R, Shen T, Zhu Y, Chang J, Wang H, Zhang Z, et al: EGF-reduced Wnt5a transcription induces epithelial-mesenchymal transition via Arf6-ERK signaling in gastric cancer cells. Oncotarget 6: 7244-7261, 2015.

29. Laurenzana A, Biagioni A, Bianchini F, Peppicelli S, Chillà A, Margheri F, Luciani C, Pimpinelli N, Del Rosso M, Calorini L, et al: Inhibition of uPAR-TGF $\beta$ crosstalk blocks MSC-dependent EMT in melanoma cells. J Mol Med 93: 783-794, 2015.

30. Clapéron A, Mergey M, Nguyen Ho-Bouldoires TH, Vignjevic D, Wendum D, Chrétien Y, Merabtene F, Frazao A, Paradis V, Housset C, et al: EGF/EGFR axis contributes to the progression of cholangiocarcinoma through the induction of an epithelial-mesenchymal transition. J Hepatol 61: 325-332, 2014.

31. Grassi ML, Palma CS, Thomé CH, Lanfredi GP, Poersch A and Faça VM: Proteomic analysis of ovarian cancer cells during epithelial-mesenchymal transition (EMT) induced by epidermal growth factor (EGF) reveals mechanisms of cell cycle control. J Proteomics 151: 2-11, 2017.

32. Xu Q, Zhang Q, Ishida Y, Hajjar S, Tang X, Shi H, Dang CV and Le AD: EGF induces epithelial-mesenchymal transition and cancer stem-like cell properties in human oral cancer cells via promoting Warburg effect. Oncotarget 8: 9557-9571, 2017.

33. Han J, Xie Y, Lan F, Yu Y, Liu W, Chen J, Zheng F, Ouyang X, Lin X, Lin Y, et al: Additive effects of EGF and IL-1 $\beta$ regulate tumor cell migration and invasion in gastric adenocarcinoma via activation of ERK1/2. Int J Oncol 45: 291-301, 2014.

34. da Rosa MR, Falcão AS, Fuzii HT, da Silva Kataoka MS, Ribeiro AL, Boccardo E, de Siqueira AS, Jaeger RG, de Jesus Viana Pinheiro J and de Melo Alves Júnior S: EGFR signaling downstream of EGF regulates migration, invasion, and MMP secretion of immortalized cells derived from human ameloblastoma. Tumour Biol 35: 11107-11120, 2014. 
35. Guo B, Gao J, Zhan J and Zhang H: Kindlin-2 interacts with and stabilizes EGFR and is required for EGF-induced breast cancer cell migration. Cancer Lett 361: 271-281, 2015.

36. Yu XX, Hu Z, Shen X, Dong LY, Zhou WZ and Hu WH: IL-33 promotes gastric cancer cell invasion and migration via ST2-ERK1/2 pathway. Dig Dis Sci 60: 1265-1272, 2015.

37. Hong H, Jiang L, Lin Y, He C, Zhu G, Du Q, Wang X, She F and Chen Y: TNF-alpha promotes lymphangiogenesis and lymphatic metastasis of gallbladder cancer through the ERK1/2/AP-1/ VEGF-D pathway. BMC Cancer 16: 240, 2016.

38. Kong B, Michalski CW, Hong X, Valkovskaya N, Rieder S, Abiatari I, Streit S, Erkan M, Esposito I, Friess H, et al: AZGP1 is a tumor suppressor in pancreatic cancer inducing mesenchymalto-epithelial transdifferentiation by inhibiting TGF- $\beta$-mediated ERK signaling. Oncogene 29: 5146-5158, 2010.

39. Shirakihara T, Horiguchi K, Miyazawa K, Ehata S, Shibata T, Morita I, Miyazono K and Saitoh M: TGF- $\beta$ regulates isoform switching of FGF receptors and epithelial-mesenchymal transition. EMBO J 30: 783-795, 2011.

40. Li Y, Shen Y, Miao Y, Luan Y, Sun B and Qiu X: Co-expression of uPAR and CXCR4 promotes tumor growth and metastasis in small cell lung cancer. Int J Clin Exp Pathol 7: 3771-3780, 2014.
41. Pavón MA, Arroyo-Solera I, Céspedes MV, Casanova I, León X and Mangues R: uPA/uPAR and SERPINE1 in head and neck cancer: Role in tumor resistance, metastasis, prognosis and therapy. Oncotarget 7: 57351-57366, 2016

42. LaRusch GA, Mahdi F, Shariat-Madar Z, Adams G, Sitrin RG, Zhang WM, McCrae KR and Schmaier AH: Factor XII stimulates ERK1/2 and Akt through uPAR, integrins, and the EGFR to initiate angiogenesis. Blood 115: 5111-5120, 2010.

43. Zheng D, Hu Z, He F, Gao C, Xu L, Zou H, Wu Z, Jiang X and Wang J: Downregulation of galectin-3 causes a decrease in uPAR levels and inhibits the proliferation, migration and invasion of hepatocellular carcinoma cells. Oncol Rep 32: 411-418, 2014.

44. Ahmed N, Oliva K, Wang Y, Quinn Mand Rice G: Downregulation of urokinase plasminogen activator receptor expression inhibits Erk signalling with concomitant suppression of invasiveness due to loss of uPAR-betal integrin complex in colon cancer cells. $\mathrm{Br}$ J Cancer 89: 374-384, 2003.

45. Raghu H, Gondi CS, Dinh DH, Gujrati M and Rao JS: Specific knockdown of uPA/uPAR attenuates invasion in glioblastoma cells and xenografts by inhibition of cleavage and trafficking of Notch-1 receptor. Mol Cancer 10: 130, 2011. 